\title{
DIVERSIDAD hAPLOTIPICA DE CINCO STR DEL CROMOSOMA Y EN LA POBLACIóN DE LIMA-PERú
}

\author{
Jorge Hau Camoretti ${ }^{1,2}$ \\ Cynthia Leon Mendoza. ${ }^{2}$
}

\begin{abstract}
RESUMEN
La tipificación molecular de ADN del cromosoma $\mathrm{Y}$ es una herramienta de reconocida importancia en el proceso de identificación de individuos de género masculino en diversos casos forenses. Actualmente es una herramienta de apoyo para los laboratorios Forenses en la identificación de víctimas en caso de violaciones y restos humanos en Lima Perú, en este estudio se determina el haplotipo del cromosoma Y, que será comparado con parientes por línea paterna de género masculino, con el fin de realizar el análisis estadístico de estos marcadores, aportara una base de datos peruana y comparar con parientes de la línea masculina.

Distribución de frecuencias alélicas y haplotípicas para los 03 marcadores analizados: se determinaron 77 haplotipos con 03 marcadores STR, y en la población de Lima Perú, realizada durante los años 2010-2012.
\end{abstract}

Palabras clave: Tipificación ADN, Cromosoma Y, Diversidad haplotipica, STR Y.

\section{SUMMARY}

The application of Y-Chromosome molecular DNA typing is a tool of recognized importance in the process of identification of male individuals in various forensic cases, and currently it is now a support tool for genetic a laboratories seeking to identify victims. In this report, the Y-chromosome haplotype is determined and statistical analyses are performed to improve data bases of Peruvian Y-chromosome for comparison with relatives of the male line.

Distributions of allele and haplotype frequencies for the 05 markers were analyzed: 77 haplotypes were determined with 05Y-STR markers inLima-Perú Population during 2010-2012.

Keywords: Typing DNA, Y chromosome, haplotipo, diversity, STR Y

\section{INTRODUCCIóN}

En Criminalística una ciencia que ha desarrollado enormemente es la Biología Molecular con el estudio del Acido Desoxirribonucleicos (ADN). Los análisis de ADN se han transformado en una de las pruebas más importante en la investigación Criminal y en los casos de paternidad discutida.

En el Perú ha aumentado los casos de violaciones sexuales y la prueba de ADN es muy importan- te para determinar a quién le pertenece el semen encontrado en la victima y en la escena del delito, para ello es necesario tener la frecuencia poblacional de los marcadores del cromosoma Y, para poder establecer la probabilidad de coincidencia entre la muestras analizadas con estadística poblacional peruana.

Los marcadores moleculares del cromosoma-Y son importantes en la identificación humana por su diversidad o polimorfismo (gran variabilidad entre

1 Laboratorio de Biología Molecular ADN Forense, Dirección de Criminalística de la Policía Nacional del Perú.

2 Laboratorio de Bioquímica y Genética Molecular, Facultad de Ciencias Biológicas Universidad Ricardo Palma. 
individuos), resultante de variaciones en la secuencia del genoma (alelos) en regiones no codificantes, determinadas por el número de veces que se presenta un alelo con respecto al número total de alelos en una población para un locus en particular (frecuencia génica), lo que refleja el grado de heterogeneidad y su poder de discriminación. El cromosoma-Y, por no recombinarse, transmite los polimorfismos en forma ligada de generación en generación en individuos de sexo masculino como un haplotipo (combinación de estados alélicas de secuencias polimórficas localizadas en el cromosoma-Y humano), y traza la evolución del linaje paternal, además refleja las migraciones humanas y permite el uso en identificación humana. ${ }^{1,2}$

En procesos de identificación por ADN, se comparan los perfiles genéticos de casos de violaciones y restos esqueléticos exhumados en varias poblaciones del Perú con los perfiles genéticos obtenidos de muestras de referencia de familiares de las personas reportadas como desaparecidas, tipificadas con marcadores STR autosómicos de rutina (AmpFiSTR ${ }^{\circledR}$ Identifiler ${ }^{\mathrm{TM}}$ de Applied Biosystems y PowerPlex16®System de Promega) o marcadores STR autosómicos utilizados para muestras en mal estado de conservación o con presencia de in hibidores(PowerPlex16HS ${ }^{\circledR}$ SystemdePromega y AmpFiSTR ${ }^{\circledR}$ Minifiler ${ }^{\mathrm{TM}}$ de Applied Biosystems). Para estos casos, las muestras de referencia de familiares en primer grado de consanguinidad (hijos y progenitores) son las más indicadas para dar una probabilidad informativa mayor a $99.9 \%$. Sin embargo, no siempre se cuenta con familiares ascendientes y descendientes directos de los desaparecidos sino que esta comparación se hace con otros familiares, quienes pueden ser hermanos paternos, abuelos paternos u otros parientes, evaluándose un vínculo de parentesco patrilineal donde se obtiene un haplotipo con marcadores STR-Y de los Kit comercial AmpFiSTR ${ }^{\circledR}$ Yfiler $^{\mathrm{TM}}$ sw Applied Biosystems y Powerplex Y System de Proimega que puede complementar el índice de parentesco y probabilidad que en algunos casos, es menor a 99.9\%. También es una herramienta útil en desastres masivos en los que no se pueden individualizar los restos mortales, ni determinar la cuarteta básica (sexo, edad, estatura y raza) como tamizaje y correlación con los grupos familiares para seguir con el proceso de identificación. Igualmente, en paternidad civil de hijos varones, con ausencia del padre biológico, el haplotipo debe coincidir con el haplotipo de parientes masculinos del presunto padre, determinando se el linaje paterno del grupo familiar. Adicionalmente es útil en los delitos contra la libertad sexual de los que se obtengan mezclas de fluidos o varios perfiles de ADN de individuos de género masculino, o bien con el fin de esclarecer otro tipo de crímenes ${ }^{3,4,5}$.

En estos estudios es fundamental el conocimiento previo de las frecuencias génicas y haplotípicas de los marcadores del cromosoma-Y de la población de Lima puesto que tiene un alto grado de mestizaje y aquí están presentes persona de los diferentes partes del Perú, para fortalecer la base de datos de referencia nacional que permita la asignación de valores de probabilidad, útiles en identificación humana ${ }^{6,7}$.

Por esta razón se presentan en este estudio los haplotipos y estimaciones de las frecuencias poblacionales resultado del análisis de 03 marcadores STRs del cromosoma-Y ( DYS437, DYS438,DYS439,) de individuos de poblaciones de Lima, con el fin de fundamentar este sistema de filiación por línea paterna para ser utilizado en una gran base de datos de referencia nacional que recopile frecuencias génicas y haplotípicas de los análisis poblacionales estudiados por elLaboratorio de Biología Molecular ADN Forense de la Dirección de Criminalística de la Policía Nacional del Perú.

Los resultados a obtenerse nos permitirán la introducción de los primeros datos del "Cromosoma Y", al Banco de Datos de ADN de la Policía Nacional del Perú, con los que se podrán conocer cual o cuales de los marcadores moleculares son más y menos polimórficos, así como también se lograra determinar la frecuencia haplotipica cada marcador molecular, la diversidad haplotípica y la capacidad de discriminación.

En la base de datos Y-STR Haplotype Referente Database, que se puede encontrar en http://ystr.charite.de/ index_gr.html, hay casi 10.000 haplotipos de 72 poblaciones distintas, y se actualiza de forma continua. Por otro lado, existe una base de datos similar para población de Estados Unidos en la página Web http:// ystr.org/usa/, con casi 2,000 haplotipos provenientes de 30 poblaciones distintas.

Una de las grandes contribuciones del presente trabajo de investigación será en la eficiente administración de justicia, donde las frecuencias haplotípicas de los marcadores moleculares del cromosoma $Y$, hallados serán empleados en los cálculos estadísticos de los dictámenes periciales de determinación de linaje paterno y en casos de asaltos sexuales. Al utilizar frecuencias propias de la población peruana 
en los cálculos estadísticos harán que se obtengan resultados sean más precisos, dejándose de lado las frecuencias de hispanos americanos o de países vecinos.

\section{MATERIAL Y MÉTODOS}

a. Muestras biológicas: se recolectaron 250 muestras de individuos varones no emparentados de 05 zonas que se dividió Lima: Centro, Sur, Norte, Este, Oeste; las muestras biológicas se extrajeron por punción del dedo y se impregnaron a las tarjetas de FTA para sangre, se rotularan y dejaran secar a temperatura ambiente ${ }^{12}$.

b. Sujetos de Experiencia: Se tomarán 250 muestras de individuos varones no emparentados, los que fueron agrupados en cinco grupos correspondientes a las zonas: este, oeste, norte, sur y centro de la ciudad de Lima, tomándoseles a cada uno como muestra biológica: sangre.

Los donantes o sujetos de experiencia fueron informados del estudio ha realizarse, para lo cual se elaboró fichas de consentimiento, en el que se plasmó la investigación ha realizarse, los propósitos de la investigación, datos de las personas, sus familiares, tipos de muestras a tomarse; las que deberán ser firmadas en señal de su respectivo consentimiento

c. Métodos a Utilizar: Una vez tomadas las muestras se secaron y guardaron a temperatura ambiente hasta su análisis en el laboratorio, donde se realizólas siguientes etapas:

Extracción de ADN: Extracción de ADN de sangre

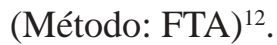

Amplificación del ADN. La amplificación del Kit PowerPlex ${ }^{\circledR}$ Y System (Manual del usuario del Promega con 03 marcadores cromosoma-Y (DYS437, DYS438, DYS439,) se realizó en un volumen final de $25 \mu \mathrm{l}$ con los siguientes componentes: Gold STR 10X Buffer 2.5 ul, PowerPlex Y 10X Primer Pair mix 2.5 ul, Amplitaq Gold DNA polymerase 0.55 ul, ADN de $1 \mathrm{nr} 19.42$ ul. La reacción en cadena de la polimerasa (PCR) se llevó a cabo en un Termociclador GeneAmp®PCR System 97009, 10,11.

Tipificación de los polimorfismos del ADN. Se utilizó un Analizador Genético ABI PRISM®310 (Applied-Biosystems, Foster City, CA) ${ }^{9}$ para tipificación molecular y se siguieron las instrucciones de la casa comercial. El software Genotyper®, analiza el estándar de tamaño de control interno Internal Lane Standard 600en cada muestra y la escalera alélica para asegurarla precisión en cada inyección del capilar. La asignación alélica se realizó por comparación de los fragmentos obtenidos en pares de bases para cada pico del alelo de las muestras analizadas con los picos de la escalera alélica PowerPlex Y, y se tuvieron en cuenta las recomendaciones del grupo de referencia de la Sociedad Internacional de Genética Forense (ISFG) ${ }^{13,10}$.

Análisis estadísticos: La estimación de las frecuencias alélicas y haplotípicas se definió como la proporción en la que se encuentra cada alelo o haplotipo dentro de la población de estudio y la frecuencia de los haplotipos. La diversidad alélica (génica) y haplotípica fue calculada para evaluar la eficacia de los marcadores moleculares usados con respecto a la sumatoria de las frecuencias de cada polimorfismo y haplotipos en la población de estudio, se usó el software Arlequín versión 3.1. La capacidad discriminatoria se determinó con el número de haplotipos observados sobre el total de individuos analizados y la probabilidad de coincidencia al azar se estimó para determinarla probabilidad quedos individuos seleccionados al azar en la población tengan un mismo haplotipo de ADN cromosoma- Y.

\section{RESULTADOS}

En la tabla $\mathrm{N}^{\circ} 01$ se observa lo siguiente:

- El Marcador DY S437 solo se encontró 04 alelos: $13,14,15,16$ con una diversidad Génica de 0.483 .

- El DY S438 solo se encontró 04 alelos : 10, 11, 12, 13 con una diversidad Génica de 0.6067

- El DY S439 solo se encontró 06 alelos: 10, 11, $12,13,14.15,16$ con una diversidad Génica de 0.6310

En la tabla $\mathrm{N}^{\circ} 02$ se observa lo siguiente:

- Se obtuvo 77 haplotipos diferente entre las 250 muestras analizadas,

- Las frecuencias de los haplotipos se observa donde se encuentra más de 01 haplotipo, se ha visto que corresponde a una misma de ciudad de origen, como es el caso de haplotipo 31, se nos demuestra que en la ciudad de Lima Perú hay una conjunción de los todas las ciudades del Perú 
Tabla 1: Distribución de la frecuencia alélica, número de alelos y diversidad génica de los 03 SRT del cromosoma $\mathrm{Y}$ de la población de Lima.

\begin{tabular}{|c|c|c|c|}
\hline ALELOS & DY S437 & DY S438 & DY S439 \\
\hline 7 & & & \\
\hline 8 & & & \\
\hline 9 & & & \\
\hline 10 & & 0.115 & 0.034 \\
\hline 11 & & 0.494 & 0.241 \\
\hline 12 & & 0.379 & 0.540 \\
\hline 13 & 0.011 & 0.011 & 0.161 \\
\hline 14 & 0.667 & & 0.011 \\
\hline 15 & 0.276 & & 0.011 \\
\hline 16 & 0.046 & & \\
\hline NA & 4 & 4 & 6 \\
\hline GD & 0.4830 & 0.6067 & 0.6310 \\
\hline
\end{tabular}

NA : Numero de alelos

GD: Diversidad Génica

Tabla 2: Distribución de la frecuencia haplotípica de los 03 SRT del cromosoma y de la población de Lima.

\begin{tabular}{|c|c|c|c|c|}
\hline & DY S437 & DY S438 & DY S439 & $\mathrm{N}^{\circ}$ \\
\hline $\mathrm{H} 1$ & 14 & 12 & 12 & 5 \\
\hline $\mathrm{H} 2$ & 15 & 12 & 12 & 7 \\
\hline $\mathrm{H} 3$ & 14 & 11 & 12 & 5 \\
\hline $\mathrm{H} 4$ & 14 & 12 & 13 & 10 \\
\hline $\mathrm{H} 5$ & 14 & 11 & 12 & 1 \\
\hline H6 & 15 & 12 & 12 & 1 \\
\hline H7 & 15 & 12 & 13 & 5 \\
\hline H8 & 14 & 11 & 11 & 1 \\
\hline H9 & 15 & 11 & 12 & 1 \\
\hline H10 & 14 & 12 & 12 & 5 \\
\hline H11 & 14 & 11 & 12 & 7 \\
\hline H12 & 14 & 12 & 12 & 6 \\
\hline H13 & 14 & 12 & 12 & 9 \\
\hline H14 & 14 & 12 & 11 & 5 \\
\hline $\mathrm{H} 15$ & 14 & 11 & 12 & 11 \\
\hline H16 & 14 & 11 & 11 & $\frac{1}{2}$ \\
\hline H17 & 14 & 12 & 11 & 11 \\
\hline H18 & 15 & 12 & 12 & 1 \\
\hline H19 & 16 & 10 & 12 & $\frac{1}{4}$ \\
\hline $\mathrm{H} 20$ & 15 & 12 & 11 & \\
\hline $\mathrm{H} 21$ & 14 & 11 & 12 & 1 \\
\hline $\mathrm{H} 22$ & 14 & 11 & 10 & 6 \\
\hline $\mathrm{H} 23$ & 14 & 11 & 13 & \\
\hline H24 & 14 & 10 & 13 & 5 \\
\hline $\mathrm{H} 25$ & 14 & 12 & 12 & \\
\hline H 26 & 15 & 10 & 12 & 5 \\
\hline H 27 & 14 & 11 & 13 & 1 \\
\hline H 28 & 14 & 11 & 12 & \\
\hline Н 29 & 14 & 11 & 13 & 4 \\
\hline H 30 & 14 & 11 & 2 & \\
\hline H 31 & 14 & 11 & 3 & 15 \\
\hline 32 & 14 & 11 & 10 & \\
\hline 33 & 15 & 12 & 11 & \\
\hline H33 & 14 & 12 & 11 & \\
\hline H 34 & 14 & 10 & 11 & $\frac{1}{5}$ \\
\hline H 35 & 14 & 11 & 12 & \\
\hline H36 & 14 & 11 & 13 & \\
\hline H 37 & 14 & 11 & 12 & 11 \\
\hline H 38 & 15 & 12 & 11 & 5 \\
\hline H 39 & 14 & $\frac{12}{10}$ & $\frac{11}{10}$ & \\
\hline H 40 & 16 & 11 & 12 & \\
\hline H 41 & $\frac{10}{14}$ & $\frac{11}{11}$ & 11 & \\
\hline H 42 & 15 & 12 & 12 & 5 \\
\hline H 43 & 15 & 11 & 12 & \\
\hline H 44 & 14 & $\frac{11}{10}$ & & \\
\hline H45 & 14 & 12 & 12 & \\
\hline H 46 & 14 & 11 & 11 & 2 \\
\hline H 47 & 15 & 12 & 12 & 5 \\
\hline & DY S437 & DY S438 & DY S439 & $\frac{N^{\circ}}{N^{\circ}}$ \\
\hline
\end{tabular}

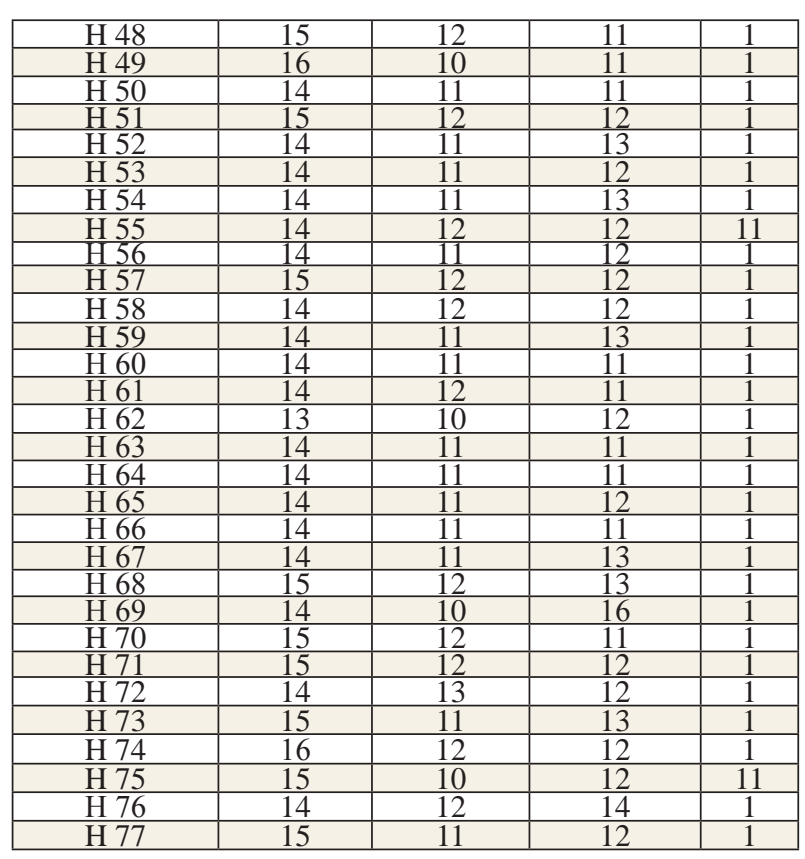

\section{REFERENCIA BIBLIOGRÁFICA}

1. ANDRADAS J. 1995. El Análisis del ADN en los Vestigios Biológicos. Editorial RVC, Madrid-España,

2. ANSLINGER K, Keil W, Weichhold G, and Eisenmenger W. 2000; Y Chromosome STR haplotypes in a population sample from Bavaria. Journal of Legal Medicine; 113:189-192.

3. BUDOWLE B., Brown BL. 2001;El Uso de ADN en la Identificación Forense. Revista Iberoamericana de Criminalística, Criminología, Medicina y Ciencias Forenses: Forénsica 1(01): 9-22.

4. BUSTAMANTE JC. 1993. Estudio Médico Legal de los Marcadores Genéticos empleados en Investigación de Paternidad y Criminalística (Tesis Doctoral). Universidad Complutense Madrid-España,

5. BRAVO AGUILAR ML. 2002; Introducción a la Genética Forense: De las pruebas de Paternidad. Editorial Universidad de Antioquia-Colombia,

6. CARRACEDO A, Barros F.1996 Problemas Bioestadísticos en Genética Forense. Edita Servicios de Publicaciones e Intercambio Científico, Campus Universitario Sur, Imprenta Universitaria, Universidad de Santiago de CompostelaEspaña, 
7. CORACH D. 1998; Curso Teórico-Práctico: "Investigación de Evidencias Forenses Mediante Análisis de ADN". Servicio de Huellas Digitales Genéticas, Facultad de Farmacia y Bioquímica, Universidad de Buenos Aires, 1998.

8. GILL P., Brenner C, Brinkmann B, Budowle B, Carracedo A, et al.2001; DNA Commission of the International Society of Forensic Genetics: recommendations on forensic analysis using Ychromosome short tandem repeats. Legal Medicine; $3: 252-7$

9. PE APPLIED BIOSYSTEMS. 2005; ABI PRISM ${ }^{\mathrm{TM}}$ 310 Genetic Analyzer, User's Manual, PE Applied
Biosystems. A Division of Perkin Elmer, the Perkin Elmer Corporation-USA,

10.PROMEGA CORPORATION. Technical Manual $\mathrm{N}^{\circ}$ DO18 de PowerPlex Y System, USA, pp 1-37.

11.PROMEGACORPORATION.1997; Proccedings from the first European Symposium on Human Identification,

12.WHATMAN BIOSCIENCE. Manual FTA Technology: collect, transport, archive and purify nucleic acid. WB 120047, 1999. 